https://doi.org/10.15407/dopovidi2019.07.009

UDC 517.5

\author{
V.Ya. Gutlyanskii ${ }^{1}$, O.V. Nesmelova ${ }^{1,3}$, V.I. Ryazanov ${ }^{1,2}$ \\ ${ }^{1}$ Institute of Applied Mathematics and Mechanics of the NAS of Ukraine, Slov'yansk \\ ${ }^{2}$ Bogdan Khmelnytsky National University of Cherkasy \\ ${ }^{3}$ Donbas State Pedagogical University, Slov'yansk \\ E-mail: vgutlyanskii@gmail.com, star-o@ukr.net,vl.ryazanov1@gmail.com
}

\title{
On semilinear equations in the complex plane
}

Presented by Corresponding Member of the NAS of Ukraine V.Ya. Gutlyanskĭ

We study the Dirichlet problem for the semilinear partial differential equations $\operatorname{div}(A \nabla u)=f(u)$ in simply connected domains $D$ of the complex plane $\mathbb{C}$ with continuous boundary data. We prove the existence of the weak solutions $u$ in the class $C \cap W_{\mathrm{loc}}^{1,2}(D)$, if a Jordan domain D satisfies the quasihyperbolic boundary condition by Gehring-Martio. An example of such a domain that fails to satisfy the standard (A)-condition by Ladyzhenskaya-Ural'tseva and the known outer cone condition is given. Some applications of the results to various processes of diffusion and absorption in anisotropic and inhomogeneous media are presented.

Keywords: Dirichlet problem, semilinear elliptic equations, conformal and quasiconformal mappings, anisotropic and inhomogeneous media.

1. Introduction. In our last paper [1], we have established the existence of regular solutions of the Dirichlet problem for the quasilinear Poisson equation

$$
\Delta U(z)=h(z) \cdot f(U(z))
$$

in the unit disk $\mathbb{D}=\{z \in \mathbb{C}:|z|<1\}$ with continuous boundary values. We assumed that $h: \mathbb{D} \rightarrow \mathbb{R}$ is in $L^{p}(\mathbb{D}), p>1$, and $f: \mathbb{R} \rightarrow \mathbb{R}$ is continuous and $f(t) / t \rightarrow 0$ as $t \rightarrow \infty$.

This result and the theory of quasiconformal mappings (see, e.g., [2]), give a base for the study of the semilinear equations

$$
\operatorname{div}[A(z) \nabla u(z)]=f(u(z))
$$

describing many physical phenomena in anisotropic and inhomogeneous media.

Given a simply connected domain $D$ in the complex plane $\mathbb{C}$, denote, by $M_{K}^{2 \times 2}(D)$, the class of all $2 \times 2$ symmetric matrix functions $A(z)=\left\{a_{j k}(z)\right\}$ with measurable real-valued entries and $\operatorname{det} A(z)=1$, satisfying the uniform ellipticity condition

$$
\frac{1}{K}|\xi|^{2} \leqslant\langle A(z) \xi, \xi\rangle \leqslant K|\xi|^{2} \text { a.e. in } D
$$

(C) V.Ya. Gutlyanskiı̌, O.V. Nesmelova, V.I. Ryazanov, 2019 
for every $\xi \in \mathbb{R}^{2}$, where $1 \leqslant K<\infty$.

Equations (2) are closely relevant to the so-called Beltrami equations. Let $\mu: D \rightarrow \mathbb{C}$ be a measurable function with $|\mu(z)|<1$ a.e. The equation

$$
\omega_{\bar{z}}=\mu(z) \cdot \omega_{z},
$$

where $\omega_{\bar{z}}=\left(\omega_{x}+i \omega_{y}\right) / 2, \omega_{z}=\left(\omega_{x}-i \omega_{y}\right) / 2, z=x+i y, \omega_{x}$ and $\omega_{y}$ are partial derivatives of the function $\omega$ with respect to $x$ and $y$, is said to be a Beltrami equation. Equation (4) is said to be nondegenerate, if $\|\mu\|_{\infty}<1$. Homeomorphic solutions of the nondegenerate equations (4) with the first generalized derivatives by Sobolev are called quasiconformal mappings (see, e.g., [2]).

We say that a quasiconformal mapping $\omega$ satisfying (4) is agreed with $A \in M_{K}^{2 \times 2}(D)$, if

$$
\mu(z)=\frac{a_{22}(z)-a_{11}(z)-2 i a_{12}(z)}{\operatorname{det}(I+A(z))},
$$

where $I$ is the unit $2 \times 2$ matrix. Condition (3) is now written as

$$
|\mu(z)| \leqslant \frac{K-1}{K+1} \text { a.e. in } D \text {. }
$$

Vice versa, given a measurable function $\mu: D \rightarrow \mathbb{C}$, satisfying (6), one can invert the algebraic system (5) to obtain the matrix function $A \in M_{K}^{2 \times 2}(D)$ :

$$
A(z)=\left(\begin{array}{ll}
\frac{|1-\mu|^{2}}{1-|\mu|^{2}} & \frac{-2 \operatorname{Im} \mu}{1-|\mu|^{2}} \\
\frac{-2 \operatorname{Im} \mu}{1-|\mu|^{2}} & \frac{|1+\mu|^{2}}{1-|\mu|^{2}}
\end{array}\right) .
$$

Note that, by the known existence theorem for the Beltrami equations (see, e.g., Theorem V.1.3 in [2]), any $A \in M_{K}^{2 \times 2}(D)$ with condition (3) in a simply connected domain $D$ generates a quasiconfomal mapping $\omega: D \rightarrow \mathbb{D}$ through Eq. (4) with $\mu$ given by (5), where $\mathbb{D}$ is the unit disk in $\mathbb{C}$.

2. Some definitions and preliminary remarks. Following [3], under a weak solution of Eq. (2), we understand a function $u \in C \cap W_{\text {loc }}^{1,2}(\Omega)$ such that, for all $\eta \in C \cap W_{0}^{1,2}(D)$,

$$
\int_{D}\langle A(z) \nabla u(z), \nabla \eta(z)\rangle d m(z)+\int_{D} f(u(z)) \eta(z) d m(z)=0 .
$$

A fundamental role in the study of the posed problem will play the following factorization theorem (see, e.g., [4], Theorem 1, or [3], Theorem 4.1). A function $u: D \rightarrow \mathbb{R}$ is a weak solution of (2) in the class $C \cap W_{\text {loc }}^{1,2}(D)$, iff $u=U \circ \omega$, where $\omega: D \rightarrow \mathbb{D}$ is a quasiconformal mapping agreed with $A$, and $U$ is a weak solution in the class $C \cap W_{\text {loc }}^{1,2}(\mathbb{D})$ of the quasilinear Poisson equation

$$
\Delta U(w)=J(w) \cdot f(U(w)), \quad w \in \mathbb{D},
$$


$J$ denotes the Jacobian of the inverse quasiconformal mapping $\omega^{-1}: \mathbb{D} \rightarrow D$.

The regularity properties of solutions of Eq. (9) strongly depends on the degree of integrability of $J(\omega)$. Note that the mapping $\omega^{*}:=\omega^{-1}$ is extended to a quasiconformal mapping of $\mathbb{C}$ onto itself, if $\partial D$ is the so-called quasicircle (see, e.g., Theorem II.8.3 in [2]). The well-known Bojarski result (see, e.g., [5]) says that the generalized derivatives of a quasiconformal mapping in the plane are locally integrable with some power $q>2$. Note also that the Jacobian $J(w)=\left|\omega_{w}^{*}\right|^{2}-\left|\omega_{\bar{w}}^{*}\right|^{2}$. Consequently, in this case, $J \in L^{p}(\mathbb{D})$ for some $p>1$.

Recall that the image of the unit disk $\mathbb{D}$ under a quasiconformal mapping of $\mathbb{C}$ onto itself is called a quasidisk and its boundary is called a quasicircle or a quasiconformal curve. Recall also that a Jordan curve is a continuous one-to-one image of the unit circle in $\mathbb{C}$. As known, such a smooth $\left(C^{1}\right)$ or Lipschitz curve is a quasiconformal curve and, at the same time, quasiconformal curves can be even locally non-rectifiable, as it follows from the well-known Van Koch snowflake example (see, e.g., point II.8.10 in [2]).

By Theorem 4.7 in [6], cf. also Theorem 1 and Corollary in [7], the Jacobian of a quasiconformal homeomorphism $\omega^{*}: \mathbb{D} \rightarrow D$ is in $L^{p}(\mathbb{D}), p>1$, iff $D$ satisfies the quasihyperbolic boundary condition by Gehring-Martio (see [8]), i.e.

$$
k_{D}\left(z, z_{0}\right) \leqslant a \cdot \ln \frac{d\left(z_{0}, \partial D\right)}{d(z, \partial D)}+b \quad \forall z \in D
$$

for some constants $a$ and $b$ and a fixed point $z_{0} \in D$, where $k_{D}\left(z, z_{0}\right)$ is the quasihyperbolic distance between the points $z$ and $z_{0}$ in the domain $D$,

$$
k_{D}\left(z, z_{0}\right):=\inf _{\gamma} \int_{\gamma} \frac{d s}{d(\zeta, \partial D)} .
$$

Here, $d(\zeta, \partial D)$ denotes the Euclidean distance from a point $\zeta \in D$ to the boundary of $D$ and the infimum is taken over all rectifiable curves $\gamma$ joining the points $z$ and $z_{0}$ in $D$.

Recall that a domain $D$ in $\mathbb{R}^{n}, n \geqslant 2$, is called satisfying $(A)$-condition, if

$$
\text { mes } D \cap B(\zeta, \rho) \leqslant \Theta_{0} \cdot \operatorname{mes} B(\zeta, \rho) \quad \forall \zeta \in \partial D, \quad \rho \leqslant \rho_{0},
$$

for some $\Theta_{0}$ and $\rho_{0} \in(0,1)$ (see 1.1.3 in [9]). Recall also that a domain $D$ in $\mathbb{R}^{n}, n \geqslant 2$, is said to satisfy the outer cone condition, if there is a cone that makes possible to be touched by its top to every boundary point of $D$ from the completion of $D$ after its suitable rotation and shift. It is clear that the outer cone condition implies (A)-condition.

Remark 1 . Note that the quasidisks $D$ satisfy (A)-condition. Indeed, the quasidisks are the so-called QED-domains by Gehring-Martio (see, Theorem 2.22 in [10]), and the latter satisfy the condition

$$
\operatorname{mes} D \cap B(\zeta, \rho) \leqslant \Theta_{*} \cdot \operatorname{mes} B(\zeta, \rho) \quad \forall \zeta \in \partial D, \quad \rho \leqslant \operatorname{dia} D
$$

for some $\Theta_{*} \in(0,1)$ (see, Lemma 2.13 in [10]), and the quasidisks (as domains with quasihyperbolic boundary) have boundaries of the zero Lebesgue measure (see, e.g., Theorem 2.4 in [6]). 
Thus, it remains to note that, by definition, the completions of quasidisks $D$ in the the extended complex plane $\overline{\mathbb{C}}:=\mathbb{C} \cup\{\infty\}$ are also quasidisks up to the inversion with respect to a circle in $D$.

Probably, the first example of a simply connected plane domain $D$ with the quasihyperbolic boundary condition, which is not a quasidisk, was constructed in [7], Theorem 2. However, this domain satisfieds (A)-condition. Probably, one of the simplest examples of a domain $D$ with the quasihyperbolic boundary condition and without (A)-condition is the union of 3 open disks with the radius 1 centered at the points 0 and $1 \pm i$. It is clear that the domain has zero interior angle at its boundary point 1 and, consequently, by Remark 1, it is not a quasidisk.

\section{Dirichlet problem for semilinear equations.}

Theorem 1. Let $D$ be a Jordan domain in $\mathbb{C}$ satisfying the quasihyperbolic boundary condition, $A \in M_{K}^{2 \times 2}(D)$, let $\varphi: \partial D \rightarrow \mathbb{R}$ be a continuous function, and let $f: \mathbb{R} \rightarrow \mathbb{R}$ be a continuous function such that

$$
\lim _{t \rightarrow \infty} \frac{f(t)}{t}=0
$$

Then there is a weak solution $u: D \rightarrow \mathbb{R}$ of Eq. (2), which is locally Hölder-continuous in $D$ and continuous in $\bar{D}$ with $\left.u\right|_{\partial D}=\varphi$. If, in addition, $\varphi$ is Hölder-continuous, then u is Hölder-continuous in $\bar{D}$.

Proof. By Theorem 1 in [4] (see, also Theorem 4.1 in [3]), if $u$ is a weak solution of (2), then $u=U \circ \omega$, where $\omega$ is a quasiconformal mapping of $D$ onto the unit disk $\mathbb{D}$ agreed with $A$, and $U$ is a weak solution of Eq. (9) with $h=J$, where $J$ stands for the Jacobian of $\omega^{-1}$. It is also easy to see that if $U$ is a weak solution of (9) with $h=J$, then $u=U \circ \omega$ is a weak solution of (2). This allows us to reduce the Dirichlet problem for Eq. (2) with a continuous boundary function $\varphi$ in the simply connected Jordan domain $D$ to the Dirichlet problem for Eq. (9) in the unit disk $\mathbb{D}$ with the continuous boundary function $\psi=\varphi \circ \omega^{-1}$. Indeed, $\omega$ is extended to a homeomorphism of $\bar{D}$ onto $\overline{\mathbb{D}}$ (see, e.g., Theorem I.8.2 in [2]). Thus, the function $\psi$ is well defined and really is continuous on the unit circle.

It is well-known that the quasiconformal mapping $\omega$ is locally Hölder-continuous in $D$ (see Theorem 3.5 in [5]). Taking into account that $D$ is a Jordan domain in $\mathbb{C}$ satisfying a quasihyperbolic boundary condition, we can show that both mappings $\omega$ and $\omega^{-1}$ are Hölder-continuous in $\bar{D}$ and $\overline{\mathbb{D}}$, correspondingly. Indeed, $\omega=H \circ \Omega$, where $\Omega$ is a conformal (Riemann) mapping of $D$ onto $\mathbb{D}$, and $H$ is a quasiconformal mapping of $\mathbb{D}$ onto itself. The mappings $\Omega$ and $\Omega^{-1}$ are Hölder-continuous in $\bar{D}$ and in $\overline{\mathbb{D}}$, correspondingly, by Theorem 1 and its corollary in [7]. Next, by the reflection principle, $H$ can be extended to a quasiconformal mapping of $\mathbb{C}$ onto itself (see, e.g., I.8.4 in [2]), and, consequently, $H$ and $H^{-1}$ are also Hölder-continuous in $\overline{\mathbb{D}}$ (see again Theorem 3.5 in [5]). Thus, the Hölder continuity of $\omega$ and $\omega^{-1}$ in closed domains follows immediately.

Finally, it is easy to see that if $\varphi$ is Hölder-continuous, then $\psi$ is also so, and all the conclusions of Theorem 1 follow from Theorem 3 in [1].

Remark 2. In Theorem 3 of [1], we assumed additionally that $|f|$ is nondecreasing with respect to $t$. However, setting $f_{*}(s)=\max _{|t| \leqslant s}|f(t)|, s \in \mathbb{R}^{+}:=[0, \infty)$, we see that the function $f_{*}: \mathbb{R}^{+} \rightarrow \mathbb{R}^{+}$is continuous and nondecreasing. Moreover, $f_{*}(s) / s \rightarrow 0$ as $s \rightarrow \infty$ by (14). Hence, all estimates in the proof of Theorem 3 in [1] remain valid without this additional condition after the change $f \rightarrow f_{*}$. 
Corollary 1. In particular, under the hypotheses of Theorem 1 on D, $\varphi$, and $f$, there is a weak solution $U$ of the quasilinear Poisson equation

$$
\Delta U(z)=f(U(z)) \text { for a.e. } z \in D
$$

which is locally Hölder-continuous in $D$ and continuous in $\bar{D}$ with $\left.U\right|_{\partial D}=\varphi$. If, in addition, $\varphi$ is Hölder-continuous, then $U$ is Hölder-continuous in $\bar{D}$.

4. Some applied corollaries. The interest in this subject is well known both from a purely theoretical point of view, due to its deep relations to linear and nonlinear harmonic analysis, and because of numerous applications of equations of this type in various areas of physics, differential geometry, logistic problems, etc. (see, e.g., [11], [12], and the references therein). In particular, the excellent book by M. Marcus and L. Veron [12] contains a comprehensive analysis of the Dirichlet problem for the semilinear equation

$$
\Delta u(z)=f(z, u(z))
$$

in smooth ( $C^{2}$ ) domains $D$ in $\mathbb{R}^{n}, n \geqslant 3$, with boundary data in $L^{1}$. Here, $t \rightarrow f(\cdot, t)$ is a continuous mapping from $\mathbb{R}$ to a weighted Lebesgue space $L^{1}(D, \rho)$, and $z \rightarrow f(z, \cdot)$ is a nondecreasing function for every $z \in D, f(z, 0) \equiv 0$, with

$$
\lim _{t \rightarrow \infty} \frac{f(z, t)}{t}=\infty .
$$

uniformly with respect to the parameter $z$ in compact subsets of $D$.

The mathematical modeling of some reaction-diffusion problems leads to the study of the corresponding Dirichlet problem for Eq. (1) with specified right-hand side. Following [13], a nonlinear system can be obtained for the density $u$ and the temperature $T$ of a reactant. Upon eliminating $T$, the system can be reduced to a scalar problem for the concentration

$$
\Delta u=\lambda \cdot f(u),
$$

where $\lambda$ stands for a positive constant.

It turns out that the reactant density $u$ may be zero in a closed interior region $D_{0}$ called $a$ dead core. If, for instance, in Eq. (18), $f(u)=u^{q}, q>0$, a particularization of the results in Chapter 1 of [11] shows that a dead core may exist, if and only if $0<q<1$ and $\lambda$ is large enough. See also the corresponding examples of dead cores in [3]. We have, by Theorem 1, the following:

Theorem 2. Let $D$ be a Jordan domain in $\mathbb{C}$ satisfying the quasihyperbolic boundary condition, $A \in M_{K}^{2 \times 2}(D), \varphi: \partial D \rightarrow \mathbb{R}$ be a continuous function. Then there is a weak solution $u: D \rightarrow \mathbb{R}$ of the semilinear equation

$$
\operatorname{div}[A(z) \nabla u(z)]=u^{q}(z), \quad 0<q<1
$$

which is locally Hölder-continuous in $D$ and continuous in $\bar{D}$ with $\left.u\right|_{\partial D}=\varphi$. If, in addition, $\varphi$ is Hölder-continuous, then $u$ is Hölder-continuous in $\bar{D}$.

We have also the following consequence of Corollary 1. 
Corollary 2. Let $D$ be a smooth Jordan domain in $\mathbb{C}$, and let $\varphi: \partial D \rightarrow \mathbb{R}$ be a continuous function. Then there is a weak solution $U$ of the quasilinear Poisson equation

$$
\Delta U(z)=U^{q}(z), \quad 0<q<1,
$$

which is continuous in $\bar{D}$ with $\left.U\right|_{\partial D}=\varphi$ and $U \cap C_{\mathrm{loc}}^{1, \alpha}(D)$ for all $\alpha \in(0,1)$. If, in addition, $\varphi$ is Hölder-continuous with some order $\beta \in(0,1)$, then $U$ is also Hölder-continuous in $\bar{D}$ with the same order.

Recall also that certain mathematical models of a heated plasma lead to nonlinear equations of the type (18). Indeed, it is known that some of them have the form $\Delta \psi(u)=f(u)$ with $\psi^{\prime}(0)=+\infty$ and $\psi^{\prime}(u)>0$, if $u \neq 0$, as, for instance, $\psi(u)=|u|^{q-1} u$ under $0<q<1$ (see, e.g., [14] and [11, p. 4]). With the replacement of the function $U=\psi(u)=|u|^{q} \cdot \operatorname{sign} u$, we have that $u=|U|^{Q} \cdot \operatorname{sign} U, Q=1 / q$, and, with the choice $f(u)=|u|^{q^{2}} \cdot \operatorname{sign} u$, we come to the equation $\Delta U=|U|^{q} \cdot \operatorname{sign} U=\psi(U)$.

Corollary 3. Let $D$ be a smooth Jordan domain in $\mathbb{C}$, and let $\varphi: \partial D \rightarrow \mathbb{R}$ be a continuous function. Then there is a weak solution $U$ of the quasilinear Poisson equation

$$
\Delta U(z)=|U(z)|^{q-1} U(z), \quad 0<q<1
$$

which is continuous in $\bar{D}$ with $\left.U\right|_{\partial D}=\varphi$ and $U \cap C_{\mathrm{loc}}^{1, \alpha}(D)$ for all $\alpha \in(0,1)$. If, in addition, $\varphi$ is Hölder-continuous with some order $\beta \in(0,1)$, then $U$ is also Hölder-continuous in $\bar{D}$ with the same order.

In the combustion theory, the following model equation

$$
\frac{\partial u(z, t)}{\partial t}=\frac{1}{\delta} \cdot \Delta u+e^{u}, \quad t \geqslant 0, \quad z \in D,
$$

occupies a special place (see, e.g., [15] and the references therein). Here, $u \geqslant 0$ is the temperature of the medium, and $\delta$ is a certain positive parameter. We restrict ourselves by stationary solutions of (22) and generalizations in anisotropic and inhomogeneous media, although our approach makes it possible to consider the parabolic case as well (see [3]). Namely, by Theorem 1, we have:

Theorem 3. Let $D$ be a Jordan domain in $\mathbb{C}$ satisfying the quasihyperbolic boundary condition, $A \in M_{K}^{2 \times 2}(D)$, and let $\varphi: \partial D \rightarrow \mathbb{R}$ be a continuous function. Then there is a weak solution $u: D \rightarrow \mathbb{R}$ of the semilinear equation

$$
\operatorname{div}[A(z) \nabla U(z)]=\delta \cdot e^{-U(z)}
$$

which is locally Hölder-continuous in $D$ and continuous in $\bar{D}$ with $\left.u\right|_{\partial D}=\varphi$. If, in addition, $\varphi$ is Hölder-continuous, then $u$ is Hölder-continuous in $\bar{D}$.

Finally, we obtain the following consequence of Corollary 1.

Corollary 4. Let $D$ be a smooth Jordan domain in $\mathbb{C}$, and let $\varphi: \partial D \rightarrow \mathbb{R}$ be a continuous function. Then there is a weak solution $U$ of the quasilinear Poisson equation

$$
\Delta U(z)=\delta \cdot e^{U(z)}
$$


which is continuous in $\bar{D}$ with $\left.U\right|_{\partial D}=\varphi$ and $U \cap C_{\mathrm{loc}}^{1, \alpha}(D)$ for all $\alpha \in(0,1)$. If, in addition, $\varphi$ is Hölder-continuous with some order $\beta \in(0,1)$, then $U$ is also Hölder-continuous in $\bar{D}$ with the same order.

This work was partially supported by grants of Ministry of Education and Science of Ukraine, project number is $0119 U 100421$.

\section{REFERENCES}

1. Gutlyanskii, V. Ya., Nesmelova, O. V. \& Ryazanov, V. I. (2018). On the regularity of solutions of quasilinear Poisson equations. Dopov. Nac. Akad. Nauk. Ukr., No. 10, pp. 9-17. https://doi.org/10.15407/dopovidi 2018.10.009

2. Lehto, O. \& Virtanen, K. I. (1973). Quasiconformal mappings in the plane, 2nd ed. Berlin, Heidelberg, New York: Springer.

3. Gutlyanskii, V. Ya., Nesmelova, O. V. \& Ryazanov, V. I. (2018). On quasiconformal maps and semilinear equations in the plane. J. Math. Sci., 229, No. 1, pp. 7-29.

4. Gutlyanskii, V. Ya., Nesmelova, O. V. \& Ryazanov, V. I. (2018). Semilinear equations in the plane with measurable data. Dopov. Nac. Akad. Nauk Ukr., No. 2, pp. 12-18. https://doi.org/10.15407/dopovidi 2018.02.012

5. Bojarski, B. V. (2009). Generalized solutions of a system of differential equations of the first order and elliptic type with discontinuous coefficients. Report Dept. Math. Stat. (Vol. 118). Jyväskylä: Univ. of Jyväskylä.

6. Astala, K. \& Koskela, P. (1991). Quasiconformal mappings and global integrability of the derivative. J. Anal. Math, 57, pp. 203-220.

7. Becker, J. \& Pommerenke, Ch. (1982). Hölder continuity of conformal mappings and nonquasiconformal Jordan curves. Comment. Math. Helv., 57, No. 2, pp. 221-225.

8. Gehring, F. W. \& Martio, O. (1985). Lipschitz classes and quasiconformal mappings. Ann. Acad. Sci. Fenn. Ser. A. I. Math., 10, pp. 203-219.

9. Ladyzhenskaya, O.A. \& Ural'tseva N.N. (1968). Linear and quasilinear elliptic equations. New York, London: Academic Press.

10. Gehring, F. W. \& Martio, O. (1985). Quasiextremal distance domains and extension of quasiconformal mappings. J. Anal. Math., 45, pp. 181-206.

11. Diaz, J.I. (1985). Nonlinear partial differential equations and free boundaries. (Vol. 1). Elliptic equations. Research Notes in Mathematics, (Vol. 106). Boston: Pitman.

12. Marcus, M. \& Veron, L. (2014). Nonlinear second order elliptic equations involving measures. De Gruyter Series in Nonlinear Analysis and Applications. (Vol. 21). Berlin: De Gruyter.

13. Aris, R. (1975).The mathematical theory of diffusion and reaction in permeable catalysts. (Vol. 1, 2). Oxford: Clarendon Press.

14. Bear, J. (1972). Dynamics of fluids in porous media. New York: Elsevier.

15. Pokhozhaev, S. I. (2010). On an equation of combustion theory. Math. Notes, 88, No. 1-2, pp. 48-56.

Received 26.03.2019

\section{В.Я. Гутлянський ${ }^{1}$,}

О.В. Несмєлова ${ }^{1,3}$, В.І. Рязанов ${ }^{1,2}$

${ }^{1}$ Інститут прикладної математики і механіки НАН України, Слов'янськ

2 Черкаський національний університет ім. Богдана Хмельницького

3 Донбаський державний педагогічний університет, Слов'янськ

E-mail: vgutlyanskii@gmail.com, star-o@ukr.net,vl.ryazanov1@gmail.com

\section{ПРО НАПІВЛІНІЙНІ РІВНЯННЯ В КОМПЛЕКСНІЙ ПЛОЩИНІ}

Досліджено задачу Діріхле для напівлінійних рівнянь в частинних похідних $\operatorname{div}(A \nabla u)=f(u)$ в однозв'язних областях $D$ комплексної площини $\mathbb{C}$ з неперервними граничними умовами. Доведено існування слабких розв'язків $u$ у класі $C \cap W_{\mathrm{loc}}^{1,2}(D)$, якщо Жорданова область $D$ задовольняє квазігіперболічну гра- 
ничну умову Герінга-Мартіо. Наведено приклад такої області, яка не задовольняє стандартну (А)-умову Ладиженської-Уральцевої та відому умову зовнішнього конуса. Також наведено деякі застосування отриманих результатів до різних процесів дифузії та поглинання в анізотропних і неоднорідних середовищах.

Ключові слова: задача Діріхле, напівлінійні еліптичні рівняння, конформні та квазіконформні відображення, анізотропні та неоднорідні середовища.

\title{
В.Я. Гутлянский ${ }^{1}$,
}

\author{
О.В. Несмелова ${ }^{1,3}$, В.И. Рязанов ${ }^{1,2}$
}

${ }^{1}$ Институт прикладной математики и механики НАН Украины, Славянск

2 Черкасский национальный университет им. Богдана Хмельницкого

3 Донбасский государственный педагогический университет, Славянск E-mail: vgutlyanskii@gmail.com, star-o@ukr.net,vl.ryazanov1@gmail.com

\section{О ПОЛУЛИНЕЙНЫХ УРАВНЕНИЯХ НА КОМПЛЕКСНОЙ ПЛОСКОСТИ}

Исследована задача Дирихле для полулинейных уравнений в частных производных $\operatorname{div}(A \nabla u)=f(u)$ в односвязных областях $D$ комплексной плоскости $\mathbb{C}$ с непрерывными граничными условиями. Доказано существование слабых решений $u$ в классе $C \cap W_{\mathrm{loc}}^{1,2}(D)$, если Жорданова область удовлетворяет квазигиперболическому граничному условию Геринга-Мартио. Приведен пример такой области, которая не удовлетворяет стандартному (А)-условию Ладыженской-Уральцевой и известному условию внешнего конуса. Также приведены некоторые применения полученных результатов к различным процессам диффузии и поглощения в анизотропных и неоднородных средах.

Ключевые слова: задача Дирихле, полулинейные эллиптические уравнения, конформные и квазиконформные отражения, анизотропные и неоднородные среды. 